PROCEEDINGS OF THE

AMERICAN MATHEMATICAL SOCIETY

Volume 126, Number 12, December 1998, Pages 3755-3762

S 0002-9939(98)04506-7

\title{
AN ORTHOGONAL FAMILY OF POLYNOMIALS ON THE GENERALIZED UNIT DISK AND LADDER REPRESENTATIONS OF $U(p, q)$
}

\author{
JOHN D. LORCH
}

(Communicated by Roe Goodman)

\begin{abstract}
Inner product structures are given for realizations of the positive spin ladder representations over the generalized unit disk $\mathbf{D}_{p, q}=U(p, q) / K$. This is accomplished by combining previous results of the author with the construction of a family of holomorphic polynomials on $\mathbf{D}_{p, q}$. These polynomials, which play a crucial role in the present work, are shown to be orthogonal with respect to Lebesgue measure, and their norms are computed. The orthogonal family is then used to invert a certain integral transform, giving the desired inner product structures.
\end{abstract}

\section{INTRODUCTION}

The postive spin ladder representations of $U(p, q)$, which occur naturally on a Fock space, can each be realized within a space of polynomial-valued functions on the generalized unit disk $\mathbf{D}_{p, q}=\left\{\zeta \in \mathbf{C}^{p \times q} \mid I_{q}-\zeta^{*} \zeta \gg 0\right\}$. This is achieved by an integral transform $\Phi$ constructed by Mantini in [13]. In the case $p=1$, where the generalized disk is simply the unit ball in $\mathbf{C}^{q}$, unitary structures for these polynomial-valued realizations have been constructed by inverting the transform $\Phi$ (see [11]). The purpose of this work is to generalize the methods and results of [11] in order to obtain unitary structures for all values of $p$.

A key role is played by a family of holomorphic polynomials which are constructed on $\mathbf{D}_{p, q}$. We show that the polynomials are orthogonal with respect to Lebesgue measure, and we compute their norms. This family of polynomials proves to be a natural generalization of the set of holomorphic monomials on the unit ball $\mathbf{B}^{n} \subset \mathbf{C}^{n}$, whose norms and orthogonality are well-known (see [18]). The main results concerning the polynomials are discussed in section 2, culminating in Theorem 2.4. In section 3 , the polynomials, along with the machinery developed in [11], are used to invert the integral transform $\Phi$, giving the desired inner product structures for the geometric realizations of the ladder representations.

Historically, the orthogonal polynomials of Laguerre, Hermite, Jacobi, and others have been widely applied in harmonic analysis, probability, and physics. For

Received by the editors January 2, 1997 and, in revised form, April 28, 1997.

1991 Mathematics Subject Classification. Primary 22E45, 22E70; Secondary 32L25, 32M15, 58G05, 81R05, 81R25.

Key words and phrases. Ladder representations, unitary structures, Penrose transform, generalized unit disk.

(C) 1998 American Mathematical Society 
a thorough account of the classical orthogonal polynomials, see [19] or [3]. As opposed to the classical case, we analyze polynomials in the complex domain $\mathbf{D}_{p, q}$, which parameterizes the homogeneous space $U(p, q) /(U(p) \times U(q))$. In general, homogeneous spaces of the form $G / K$, where $G$ is a semisimple Lie group and $K$ is a maximal compact subgroup of $G$, have been of interest to representation theorists due to the rich collection of representations of $G$ that occur as sections of a homogeneous, holomorphic vector bundle over $G / K$ (for example, see [7], [15], [16], or [1]). As a result, a substantial body of work has been devoted to the geometry and analysis of such spaces, as in [9], [17], [8], and [6]. Of particular interest to us are the results of Faraut and Koranyi in [6], where norms of spherical vectors on $G / K$ are computed. In a forthcoming paper concerning geometric realizations of representations of $G=U(p, q)$, the author will use the orthogonal family presented in this paper in conjunction with the results of [6] to compute the norms of certain highest weight vectors (see [12]).

The author would like to thank L. Mantini and E. Dunne for numerous helpful conversations.

\section{A family of orthogonal functions on $\mathbf{D}_{p, q}$}

Here we present a certain family of polynomials on the generalized unit disk $\mathbf{D}_{p, q}$ that are orthogonal with respect to Lebesgue measure. The orthogonal family is composed of basis elements for certain irreducible components of a natural action of $G L(p, \mathbf{C}) \times G L(q, \mathbf{C})$ on the space $\mathcal{P}\left(\mathbf{D}_{p, q}\right)$ of holomorphic polynomials over $\mathbf{D}_{p, q}$. In the special case $p=1$, the orthogonal family consists of holomorphic monomials (see [11]).

2.1. The orthogonal family. The generalized unit disk, on which the desired polynomials are defined, is given by

$$
\mathbf{D}_{p, q}:=\left\{\zeta \in \mathbf{C}^{p \times q} \mid I_{q}-\zeta^{*} \zeta \gg 0\right\} .
$$

The domain $\mathbf{D}_{p, q}$ is bounded in $\mathbf{C}^{p \times q}$, and is naturally diffeomorphic to $U(p, q) / K$ (see [4] or [11]). In the case $p=1, \mathbf{D}_{1, q}$ is simply the unit ball in $\mathbf{C}^{q}$.

Notation 2.1. (a) For $r, s, m \in \mathbf{N}$, let $\mathbf{N}_{0}^{r}$ and $\mathbf{N}_{0}^{r \times s}$ denote the set of $r$-tuples of nonnegative integers and $r \times s$ matrices of nonnegative integers, respectively, and let $\mathbf{N}_{0}^{r}(m)$ denote the elements $\left(j_{1}, j_{2}, \ldots, j_{r}\right)$ of $\mathbf{N}_{0}^{r}$ such that $j_{1}+j_{2}+\cdots+j_{r}=m$.

(b) For $\alpha \in \mathbf{N}_{0}^{p}$ and $\rho \in \mathbf{N}_{0}^{q}$, let

$$
M^{p \times q}(\rho, \alpha)=\left\{\gamma \in \mathbf{N}_{0}^{p \times q} \mid c(\gamma)=\alpha \text { and } r(\gamma)=\rho\right\},
$$

where $c(\gamma)$ and $r(\gamma)$ denote the column and row sums of $\gamma$.

(c) If $\gamma \in \mathbf{N}_{0}^{p \times q}$ and $\zeta=\left(\zeta_{i j}\right) \in \mathbf{D}_{p, q}$, we write

$$
\zeta^{\gamma}=\prod_{i=1}^{p} \prod_{j=1}^{q} \zeta_{i j}^{\gamma_{i j}} \text { and } \gamma !=\prod_{i=1}^{p} \prod_{j=1}^{q} \gamma_{i j} !
$$

Notice that $M(\rho, \alpha)$ is nonempty only when $|\rho|=|\alpha|$. When $p$ and $q$ are understood, we write $M(\rho, \alpha)$ for $M^{p \times q}(\rho, \alpha)$. We are now ready to define the desired functions. 
Definition 2.2. Fix $p, q, m \in \mathbf{N}$, and suppose $\alpha \in \mathbf{N}_{0}^{p}$ and $\rho \in \mathbf{N}_{0}^{q}$ with $|\alpha|=|\rho|$. For $\zeta \in \mathbf{C}^{p \times q}$, define

$$
\varphi_{\rho \alpha}(\zeta):=\sum_{\gamma \in M(\rho, \alpha)} \frac{1}{\gamma !} \zeta^{\gamma}
$$

and put

$$
\Theta_{m}:=\left\{\varphi_{\rho \alpha} \mid \alpha \in \mathbf{N}_{0}^{p}(m), \rho \in \mathbf{N}_{0}^{q}(m)\right\} .
$$

For illustration, suppose $p=q=3$, and $m=2$. One computes the elements of $\Theta_{2}$ according to the following example. We claim that for $\rho=(1,1,0)$ and $\alpha=(1,1,0)$, $\varphi_{(1,1,0)(1,1,0)}(\zeta)=\zeta_{11} \zeta_{22}+\zeta_{21} \zeta_{12}$. To verify this, we see from Definition 2.2 that we need to compute the elements of $M((1,1,0),(1,1,0))$. Recall from Notation 2.1 that $M((1,1,0),(1,1,0))$ consists of all $3 \times 3$ matrices with nonnegative integer entries whose row sum is $(1,1,0)$ and whose column sum is $(1,1,0)$. We conclude that

$$
M((1,1,0),(1,1,0))=\left\{\left(\begin{array}{lll}
1 & 0 & 0 \\
0 & 1 & 0 \\
0 & 0 & 0
\end{array}\right),\left(\begin{array}{lll}
0 & 1 & 0 \\
1 & 0 & 0 \\
0 & 0 & 0
\end{array}\right)\right\},
$$

and hence by Definition 2.2, we have that $\varphi_{(1,1,0)(1,1,0)}(\zeta)=\zeta_{11} \zeta_{22}+\zeta_{21} \zeta_{12}$.

2.2. Integration and summation results. The major results of this section are as follows.

Proposition 2.3. The mapping of $\mathbf{C}^{p \times q}$ into $\mathbf{C}$ given by

$$
\zeta \mapsto \exp \left(\sum_{i=1}^{p} \sum_{j=1}^{q} \zeta_{i j}\right)
$$

possesses the series expansion

$$
\exp \left(\sum_{i=1}^{p} \sum_{j=1}^{q} \zeta_{i j}\right)=\sum_{m=0}^{\infty}\left(\sum_{\substack{\rho \in \mathbf{N}_{a}^{q}(m) \\ \alpha \in \mathbf{N}_{0}^{p}(m)}} \varphi_{\rho \alpha}(\zeta)\right) .
$$

Proof. Given that

$$
\exp \left(\sum_{i=1}^{p} \sum_{j=1}^{q} \zeta_{i j}\right)=\sum_{m=0}^{\infty} \frac{1}{m !}\left(\sum_{i=1}^{p} \sum_{j=1}^{q} \zeta_{i j}\right)^{m},
$$

we prove Proposition 2.3 by showing that

$$
\sum_{\substack{\rho \in \mathbf{N}_{0}^{q}(m) \\ \alpha \in \mathbf{N}_{0}^{p}(m)}} \varphi_{\rho \alpha}(\zeta)=\frac{1}{m !}\left(\sum_{i=1}^{p} \sum_{j=1}^{q} \zeta_{i j}\right)^{m}
$$


Applying Definition 2.2 and the generalized binomial theorem, we obtain

$$
\begin{aligned}
\sum_{\substack{\rho \in \mathbf{N}_{0}^{q}(m) \\
\alpha \in \mathbf{N}_{0}^{p}(m)}} \varphi_{\rho \alpha}(\zeta) & =\sum_{\substack{\rho \in \mathbf{N}_{0}^{q}(m) \\
\alpha \in \mathbf{N}_{0}^{p}(m)}} \sum_{\gamma \in M(\rho, \alpha)} \frac{\zeta^{\gamma}}{\gamma !} \\
& =\frac{1}{m !} \sum_{\substack{\gamma \in \mathbf{N}_{0}^{p \times q} \\
|\gamma|=m}} m ! \frac{\zeta^{\gamma}}{\gamma !} \\
& =\frac{1}{m !}\left(\sum_{i=1}^{p} \sum_{j=1}^{q} \zeta_{i j}\right)^{m} .
\end{aligned}
$$

Theorem 2.4. Fix $m \in \mathbf{N}_{0}$, and suppose that $\varphi_{\rho \alpha}, \varphi_{\tau \beta} \in \Theta_{m}$. Then

$$
\begin{aligned}
\int_{\mathbf{D}_{p, q}} \varphi_{\rho \alpha}(\zeta) \overline{\varphi_{\tau \beta}(\zeta)} d m(\zeta) & \\
& =\delta_{\rho, \tau} \delta_{\alpha, \beta}\left[\frac{1 ! 2 ! \cdots(p-1) ! 1 ! 2 ! \cdots(q-1) !}{1 ! 2 ! \cdots(p+q-2) !} \cdot \frac{m !}{(m+p+q-1) !} \cdot \frac{1}{\alpha ! \rho !}\right],
\end{aligned}
$$

where, given $\nu, \gamma \in N_{0}^{s}, \delta_{\nu, \gamma}=1$ if $\nu=\gamma$ and $\delta_{\nu, \gamma}=0$ otherwise.

Proof. For $\zeta=\left(\zeta_{i j}\right) \in \mathbf{D}_{p, q}$, one may show that the monomial $\zeta_{11}^{m}$ is a highest weight vector for an action $L \times R$ of $\mathbf{g l}(p, \mathbf{C}) \oplus \mathbf{g l}(q, \mathbf{C})$ on $\mathcal{P}\left(m, \mathbf{D}_{p, q}\right)$ determined by

$$
L\left(E_{i j}^{p}\right)=\sum_{l=1}^{q} \zeta_{i l} \frac{\partial}{\partial \zeta_{j l}}
$$

and

$$
R\left(E_{i j}^{q}\right)=\sum_{l=1}^{p} \zeta_{l i} \frac{\partial}{\partial \zeta_{l j}}
$$

where $E_{i j}^{r}$ is the $r \times r$ elementary matrix supported in the $i j$-th entry.

We then observe that $\Theta_{m}$ forms a basis of weight vectors (with distinct weights) of the irreducible $\mathbf{g l}(p, \mathbf{C}) \oplus \operatorname{gl}(q, \mathbf{C})$-module $V_{m}$ obtained by applying lowering operators to the highest weight vector $\zeta_{11}^{m}$. In fact, due to Theorem 4.4 of [13], we may conclude that each element of $\Theta_{m}$ is a scalar multiple of some $R^{a} L^{b}\left(\zeta_{11}^{m}\right)$, where $R^{a} L^{b}$ is the lowering operator given by

$$
R^{a} L^{b}=R^{a_{2}}\left(E_{21}^{q}\right) R^{a_{3}}\left(E_{31}^{q}\right) \cdots R^{a_{q}}\left(E_{q 1}^{q}\right) L^{b_{2}}\left(E_{21}^{p}\right) R^{b_{3}}\left(E_{31}^{p}\right) \cdots L^{b_{p}}\left(E_{p 1}^{p}\right),
$$

for $a=\left(a_{2}, \ldots, a_{q}\right) \in \mathbf{N}_{0}^{q-1}, b=\left(b_{2}, \ldots, b_{p}\right) \in \mathbf{N}_{0}^{p-1}$, and $R, L$ as in equations (2) and (3), respectively (see [10]).

Since Lebesgue measure on $\mathbf{D}_{p, q}$ is invariant under the action of $K=U(p) \times U(q)$, and the elements of $\Theta_{m}$ are all of distinct weight, we conclude that the elements of $\Theta_{m}$ are orthogonal with respect to Lebesgue measure. With this structure in place, we may inductively compute

$$
\int_{\mathbf{D}_{p, q}}\left|\varphi_{\rho \alpha}(\zeta)\right|^{2} d m(\zeta)
$$


for $\varphi_{\rho \alpha} \in \Theta_{m}$ by computing the value of

$$
\int_{\mathbf{D}_{p, q}}\left|\zeta_{11}^{m}\right|^{2} d m(\zeta)
$$

for the highest weight vector $\zeta_{11}^{m}$ and applying lowering operators.

It remains to compute the value of the integral (4). Using a theorem of Hua and certain elementary combinatorical results (see [9] and [10]), we reduce the integral in (4) to an integral over the unit ball $\mathbf{D}_{1, q}$. We then use [18] to obtain

$$
\begin{aligned}
\int_{\mathbf{D}_{p, q}} & \left|\zeta_{11}^{m}\right|^{2} d m(\zeta) \\
& =\frac{c_{p, q}}{(p-1) !} \int_{\mathbf{D}_{1, q}}\left|\zeta_{11}^{m}\right|^{2}\left(1-\left|\zeta_{11}\right|^{2}-\cdots-\left|\zeta_{1 q}\right|^{2}\right)^{p-1} d m\left(\zeta_{(1)}\right) \\
& =\frac{m ! c_{p, q}}{(m+p-1+q) !}
\end{aligned}
$$

where $\zeta_{(1)}=\left(\zeta_{11}, \ldots, \zeta_{1 q}\right)$, and $c_{p, q}=\frac{1 ! 2 ! \cdots(p-1) ! 1 ! 2 ! \cdots(q-1) !}{1 ! 2 ! \cdots(p+q-2) !}$.

\section{INVERSION FORMULA AND UNITARY STRUCTURES}

We produce an inversion formula for an integral transform $\Phi_{n}$ for each pair of natural numbers $p$ and $q$. In addition to the inversion formula, we will give an assignment of unitary structure to the image of $\Phi_{n}$ with the property that geometric realizations of the ladder representations of $U(p, q)$ will be unitary. For notation, see [11].

\subsection{An integral transform.}

Proposition 3.1 ([13]). Suppose $n \in \mathbf{N}_{0}$ and $f \in \mathcal{F}_{n}^{p, q}$. Then there is an integral transform $\Phi_{n}: \mathcal{F}_{n}^{p, q} \rightarrow \mathcal{O}\left(\mathbf{D}_{p, q}, \overline{\mathcal{P}}\left(n, \mathbf{C}^{q}\right)\right)$ given by

$$
\left(\Phi_{n} f\right)(\zeta, v)=\int_{\mathbf{C}^{q}} f(\zeta w, w) e^{v^{*} w} e^{-|w|^{2}} d m(w),
$$

where $v \in \mathbf{C}^{q}$ and $\left(\Phi_{n} f\right)(\zeta, v)$ depends holomorphically on $\zeta \in \mathbf{D}_{p, q}$.

In [13], Mantini gives a geometric construction of the positive spin ladder representations of $U(p, q)$ via the transforms $\Phi_{n}$, which carry a Fock space $\mathcal{F}_{n}^{p, q}$ of $(p, q)$-holomorphic functions on $\mathbf{C}^{p+q}$ into the set $\mathcal{O}\left(\mathbf{D}_{p, q}, \overline{\mathcal{P}}\left(n, \mathbf{C}^{q}\right)\right)$ of polynomialvalued holomorphic functions on $\mathbf{D}_{p, q}$. In general, the inner product structure on this realization has been previously unknown, as opposed to the structure on the Fock space, which is well-known (see [2] or [4]). We provide an inversion formula for $\Phi_{n}$, and then use the machinery surrounding the inversion formula to develop unitary structures for the geometric realizations of the ladder representations occuring on $\Phi_{n}\left(\mathcal{F}_{n}^{p, q}\right)$. This task has already been accomplished in the case $p=1$ (see [11]). For further details regarding $\Phi_{n}$, see [13], [14], or [10]. 
3.2. Inversion formula and unitary structures. For $\alpha \in \mathbf{N}_{0}^{p}$ and $\beta \in \mathbf{N}_{0}^{q}$ with $|\beta|-|\alpha|=n$, write $\phi_{\alpha \beta}=\Phi_{n} f_{\alpha \beta}$, where $f_{\alpha \beta}(z)=z_{R}^{\alpha} \bar{z}_{S}^{\beta}$. Applying Lemma 2.5 of [11] and Definition 2.2, we may write

$$
\phi_{\alpha \beta}(\zeta, v)=\sum_{\substack{\eta \in \mathbf{N}_{0}^{q}(n) \\ \eta \leq \beta}} \alpha ! \beta ! \varphi_{(\beta-\eta), \alpha}(\zeta) \frac{\bar{v}^{\eta}}{\eta !} .
$$

Observe from (5) and Proposition 2.3 that we have now succeeded in expressing $\phi_{\alpha \beta}$ and $\exp \left(\sum \sum \zeta_{i j}\right)$ in terms the functions $\varphi_{\rho \alpha}$, whose norms and orthogonality were established in the previous section. Hence we obtain the following extension to the $(p, q)$ case of the inversion formula for $\Phi_{n}$.

Theorem 3.2. Fix $p, q \in \mathbf{N}$ and $n \in \mathbf{N}_{0}$. Suppose that $\phi \in \Phi_{n}\left(\mathcal{F}_{n}^{p, q}\right)$ and $\phi=$ $\Phi_{n} f$, where $f \in \mathcal{F}_{n}^{p, q}$. Then for $z \in \mathbf{C}^{p, q}$,

$$
f(z)=\lim _{t \rightarrow 1^{-}} \frac{d^{p+q-1}}{d t^{p+q-1}}\left[\frac{t^{p+q-1}}{c_{p, q}} \int_{\mathbf{D}_{p, q}} \int_{\mathbf{C}^{q}}(\mathcal{L} \phi)(t \zeta, v) e^{\left(z_{R}^{T} \bar{\zeta}+v^{T}\right) \bar{z}_{S}} e^{-|v|^{2}} d m(v) d m(\zeta)\right] .
$$

Proof. The argument is analogous to the proof of Theorem 3.12 in [11]. By Proposition 2.3 and Theorem 2.4, we may use the functions $\varphi_{\rho \alpha}$ in the expansion of the exponential function, and to fill the role that the monomials play in the case $p=1$.

Now we explicitly describe a Hilbert space structure on $\Phi_{n}\left(\mathcal{F}_{n}^{p, q}\right)$ with the property that the oscillator representation $\sigma_{n}$ on $\mathcal{F}_{n}^{p, q}$ will be unitarily equivalent to the geometric realization $\omega_{n}$ via $\Phi_{n}$. Using this Hilbert space structure and certain differential operators, we then apply the results of [5] to characterize $\Phi_{n}\left(\mathcal{F}_{n}^{p, q}\right)$.

Definition 3.3. For $\phi_{1}, \phi_{2} \in \mathcal{O}\left(\mathbf{D}_{p, q}, \overline{\mathcal{P}}\left(n, \mathbf{C}^{q}\right)\right)$, define

$$
\begin{aligned}
\left(\left(\phi_{1}, \phi_{2}\right)\right)_{n} & \\
& :=\lim _{t \rightarrow 1^{-}} \frac{d^{p+q-1}}{d t^{p+q-1}}\left[\frac{t^{p+q-1}}{c_{p, q}} \int_{\mathbf{D}_{p, q}} \int_{\mathbf{C}^{q}}\left(\mathcal{L} \phi_{1}\right)(t \zeta, v) \overline{\phi_{2}(\zeta, v)} e^{-|v|^{2}} d m(v) d m(\zeta)\right] \\
& =\lim _{t \rightarrow 1^{-}} \frac{d^{p+q-1}}{d t^{p+q-1}}\left[\frac{t^{p+q-1}}{c_{p, q}} \int_{\mathbf{D}_{p, q}}\left\langle\left(\mathcal{L} \phi_{1}\right)(t \zeta, \cdot), \phi_{2}(\zeta, \cdot)\right\rangle d m(\zeta)\right],
\end{aligned}
$$

whenever the integral above converges.

For $\phi \in \mathcal{O}\left(\mathbf{D}_{p, q}, \overline{\mathcal{P}}\left(n, \mathbf{C}^{q}\right)\right)$, put $\|\phi\|_{n}^{2}:=((\phi, \phi))_{n}$. Note that on the set $\{\phi \in$ $\left.\mathcal{O}\left(\mathbf{D}_{p, q}, \overline{\mathcal{P}}\left(n, \mathbf{C}^{q}\right)\right) \mid\|\phi\|_{n}^{2}<\infty\right\}$, the form $((\cdot, \cdot))_{n}$ is Hermitian and positive semidefinite. We next examine its behavior on $\Phi_{n}\left(\mathcal{F}_{n}^{p, q}\right)$.

Theorem 3.4. Let $\phi_{1}, \phi_{2} \in \Phi_{n}\left(\mathcal{F}_{n}^{p, q}\right)$, with $\phi_{1}=\Phi_{n} f_{1}$ and $\phi_{2}=\Phi_{n} f_{2}$ for $f_{1}, f_{2} \in$ $\mathcal{F}_{n}^{p, q}$. Then

$$
\left(\left(\phi_{1}, \phi_{2}\right)\right)_{n}=\left\langle f_{1}, f_{2}\right\rangle .
$$

Hence $((\cdot, \cdot))_{n}$ defines an Hermitian inner product on $\Phi_{n}\left(\mathcal{F}_{n}^{p, q}\right)$.

Proof. One follows the proof of Theorem 4.2 in [11], using Proposition 2.3 and Theorem 2.4 to let the $\varphi_{\rho \alpha}$ 's play the role that the holomorphic monomials play in the case $p=1$. 
Corollary 3.5. The vector space $\Phi_{n}\left(\mathcal{F}_{n}^{p, q}\right)$ endowed with the inner product $((\cdot, \cdot))$ is a Hilbert space.

Corollary 3.6. The representation $\omega_{n}$ on $\Phi_{n}\left(\mathcal{F}_{n}^{p, q}\right)$ is irreducible and unitary, and is unitarily equivalent to $\sigma_{n}$ via $\Phi_{n}$.

At this point one may wonder whether the inner product $((\cdot, \cdot))_{n}$ characterizes $\Phi_{n}\left(\mathcal{F}_{n}^{p, q}\right)$. In general, this inner product is not enough to characterize $\Phi_{n}\left(\mathcal{F}_{n}^{p, q}\right)$. However, when we put the inner product together with a set of differential operators, with the aid of [5] we are able to obtain a complete characterization of $\Phi_{n}\left(\mathcal{F}_{n}^{p, q}\right)$.

Definition 3.7. Let $\mathcal{D}_{p, q}$ denote the set of differential operators consisting of all $2 \times 2$ minors of the matrix of differential operators

$$
\square_{p, q}=\left(\begin{array}{ccc}
\frac{\partial}{\partial \zeta_{11}} & \cdots & \frac{\partial}{\partial \zeta_{1 q}} \\
\vdots & & \vdots \\
\frac{\partial}{\partial \zeta_{p 1}} & \cdots & \frac{\partial}{\partial \zeta_{p q}} \\
\frac{\partial}{\partial \bar{v}_{1}} & \cdots & \frac{\partial}{\partial \bar{v}_{q}}
\end{array}\right) .
$$

In the case that $q=1$, we will say that $\mathcal{D}_{p, 1}=\emptyset$.

Definition 3.8. For $p, q \in \mathbf{N}$, let

$$
\mathcal{X}_{n}^{p, q}=\left\{\phi \in \mathcal{O}\left(\mathbf{D}_{p, q}, \bar{P}\left(n, \mathbf{C}^{q}\right)\right) \mid\|\phi\|_{n}^{2}<\infty \text { and } D \phi=0 \text { for all } D \in \mathcal{D}_{p, q}\right\} .
$$

Theorem 3.9. Fix $n \in \mathbf{N}_{0}$ and $p, q \in \mathbf{N}$. Then $\Phi_{n}\left(\mathcal{F}_{n}^{p, q}\right)=\mathcal{X}_{n}^{p, q}$.

Proof. Using Theorem 3.4 and the results of [4], we obtain $\Phi_{n}\left(\mathcal{F}_{n}^{p, q}\right) \subset \mathcal{X}_{n}^{p, q}$. An argument analogous to that in [11] gives the opposite inclusion.

\section{REFERENCES}

1. M. Atiyah and W. Schmid, A geometric construction of the discrete series for semisimple Lie groups, Invent. Math. 42 (1977), 1-62. MR 57:3310

2. V. Bargmann, On a Hilbert space of analytic functions and an associated integral transform Part I, J. Funct. Anal. 14 (1961), 187-214. MR 28:486

3. T. Chihara, An Introduction to Orthogonal Polynomials, Gordon and Breach, New York, 1978. MR 58:1979

4. M. Davidson, The harmonic representation of $U(p, q)$ and its connection with the generalized unit disk, Pacific J. Math. 129 (1987), 33-55. MR 89c:22021

5. M. Davidson, T. Enright, and R. Stanke, Differential operators and highest weight representations, Mem. Amer. Math. Soc. 455 (1991), 1-117. MR 92c:22034

6. J. Faraut and A. Koranyi, Function spaces and reproducing kernels on bounded symmetric domains, J. Funct. Anal. 88 (1990), 64-89. MR 90m:32049

7. Harish-Chandra, Representations of semisimple Lie groups V, Amer. J. Math. 78 (1956), 1-41. MR 18:490c

8. S. Helgason, Groups and Geometric Analysis, Academic Press, Orlando, 1984. MR 86c:22017

9. L. K. Hua, Harmonic Analysis of Functions of Several Complex Variables, American Mathematical Society, Providence, 1963. MR 30:2162

10. J. Lorch, Unitary structures for ladder representations of $U(p, q)$, Ph.D. thesis, Oklahoma State University, 1995.

11. J. Lorch and L. Mantini, Inversion of an integral transform and ladder representations of $U(1, q)$, Representation Theory and Harmonic Analysis: A Conference in Honor of Ray Kunze (Tuong Ton-That, ed.), Comtemp. Math. 191 (1995), 117-137. MR 96m:22022

12. J. Lorch, An integral transform and ladder representations of $U(p, q)$, Pacific J. Math., in press.

13. L. Mantini, An integral transform in $L^{2}$-cohomology for the ladder representations of $U(p, q)$, J. Funct. Anal. 60 (1985), 211-241. MR 87a:22029 
14. L. Mantini, An $L^{2}$-cohomology construction of negative spin mass zero equations for $U(p, q)$, J. Math. Anal. Appl. 136 (1988), 419-449. MR 90c:22040

15. M. Narasimhan and K. Okamoto, An analog of the Borel-Weil-Bott theorem for Hermitian symmetric pairs of non-compact type, Ann. of Math. 91 (1970), 486-511. MR 43:419

16. R. Parthasarathy, Dirac operator and the discrete series, Ann. of Math. 96 (1972), 1-30. MR 47:6945

17. I.I. Piatetskii-Shapiro, Automorphic Functions and the Geometry of the Classical Domains, Gordon and Breach, New York, 1969. MR 40:5908

18. W. Rudin, Function Theory in the Unit Ball of $\mathbf{C}^{n}$, Springer-Verlag, New York, 1980. MR 82i:32002

19. G. Szegö, Orthogonal Polynomials, American Mathematical Society, Providence, 1939. MR 1:14b

Department of Mathematics, Ball State University, Muncie, Indiana 47306 